\title{
High-quality monoenergetic proton generation by sequential radiation pressure and bubble acceleration
}

\author{
Baifei Shen, ${ }^{1, *}$ Xiaomei Zhang, ${ }^{1}$ Zhengming Sheng, ${ }^{2,3}$ M. Y. Yu, ${ }^{3,4}$ and J. Cary ${ }^{5,6}$ \\ ${ }^{1}$ State Key Laboratory of High Field Laser Physics, Shanghai Institute of Optics and Fine Mechanics, Chinese Academy of Sciences, \\ Shanghai 201800, China \\ ${ }^{2}$ Department of Physics, Shanghai Jiao Tong University, Shanghai 200240, China \\ ${ }^{3}$ Institute for Fusion Theory and Simulation, Zhejiang University, Hangzhou 310027, China \\ ${ }^{4}$ Institut für Theoretische Physik I, Ruhr-Universität Bochum, D-44780 Bochum, Germany \\ ${ }^{5}$ Department of Physics, University of Colorado, Boulder, Colorado 80309, USA \\ ${ }^{6}$ Tech-X Corporation, Boulder, Colorado 80303, USA \\ (Received 25 June 2009; published 10 December 2009)
}

\begin{abstract}
Two-dimensional particle-in cell simulation shows that protons in a small target located in an underdense high-mass plasma can be accelerated by the radiation pressure of a short circularly polarized laser pulse as well as by the wake bubble field of the laser in the background plasma. The radiation-pressure preaccelerated protons are easily trapped and accelerated stably in front of the bubble for a relatively long distance. It is found that a quasimonoenergetic proton beam of $38 \mathrm{GeV}$ peak energy and $12.6 \%$ energy spread as well as small divergence angle can be obtained with a $10^{23} \mathrm{~W} / \mathrm{cm}^{2} 18.26 \mathrm{~kJ}$ laser pulse in a tritium plasma of density $5.2 \times 10^{20} \mathrm{~cm}^{-3}$.
\end{abstract}

DOI: 10.1103/PhysRevSTAB.12.121301

PACS numbers: 52.38.Kd, 52.38.Hb, 52.65.Rr

\section{INTRODUCTION}

Particle acceleration in laser-plasma interaction is of much interest because of its promising role in realizing tabletop charge-particle accelerators [1-5]. As the power of short-pulse lasers is being increased within available technology, many novel schemes for laser acceleration of particles is being proposed [1-6]. Proton beams with energies up to $60 \mathrm{MeV}$ by target-normal sheath acceleration have been observed $[7,8]$ during irradiation of foil targets by terawatt/petawatt lasers. The proton energy depends on the temperature and density of the hot electrons that are also produced in the laser-plasma interaction. It is thus difficult to further increase the proton energy since the latter scales with the square root of the laser intensity.

To generate $\mathrm{GeV}$ protons, most proposed schemes depend on acceleration fields moving at relativistic speeds. There are several approaches. One is by radiation-pressure driven foil acceleration $[9,10]$, from which $\mathrm{GeV}$ monoenergetic protons can be obtained if the laser-pulse duration is sufficiently long [10]. However, with long interaction times many undesirable effects such as instabilities can take place during the acceleration process, even if the laser pulse itself can be well controlled [11]. Another approach is by laser wakefield acceleration in the bubble regime [1215]. The bubble, a multidimensional relativistic plasma wave, is generated when the plasma electrons are pushed away by an intense short laser pulse and eventually pulled back by the space-charge electric field as the laser pulse propagates forward. The bubble cavity contains only the

\footnotetext{
*Author to whom correspondence should be addressed. bfshen@mail.shcnc.ac.cn
}

background-plasma ions and a huge space-charge field that moves forward at relativistic speed. Recent studies of bubble-driven acceleration have shown the possibility of generating high-quality quasimonoenergetic $\mathrm{GeV}$ electron beams [12]. Together with electron injection $[13,14]$, triggering of wave, or bubble, breaking by a nanowire can lead to more stable electron acceleration [15]. Under appropriate laser and plasma conditions, the protons in the background plasma can also be trapped and accelerated in the electron-bubble structure [16]. However, the accelerated protons are usually not monoenergetic.

In this paper, an efficient scheme for producing highquality quasimonoenergetic proton bunches, namely by using a hydrogen microtarget embedded in an underdense heavier-ion background plasma, is proposed. The scheme is somewhat similar to triggering of wake-wave breaking by a nanowire [15], except that here the target ions are accelerated. In this scheme, the laser pulse creates a bubble in the background plasma as well as pushes the electrons and some of the target protons (because of the intense space-charge field generated by the expelled target electrons) of the microtarget forward. These radiation-pressure preaccelerated protons are easily trapped by the bubble field and further accelerated. Since in this scheme the accelerated protons are from the microtarget, a lower background-plasma density can be used. This leads to a smaller wake bubble moving at higher velocity, so that higher energy and density protons than that from the existing schemes can be obtained. The trapped protons are also transversely better confined, so that they can be accelerated stably for a rather longer time. Our two-dimensional (2D) particle-in-cell (PIC) simulation results show that a quasimonoenergetic proton beam with a divergence angle 
of about $\Delta \theta \sim 2 \times 10^{-2} \pi$ rad and maximum kinetic energy of about $42 \mathrm{GeV}$ and peak energy of $38 \mathrm{GeV}$ can be obtained using a $10^{23} \mathrm{~W} / \mathrm{cm}^{2}$ laser pulse and a $5.2 \times$ $10^{20} \mathrm{~cm}^{-3}$ background plasma. The number of accelerated protons with energy larger than $35 \mathrm{GeV}$ is about $6.24 \times$ $10^{9}$.

\section{SIMULATION RESULTS}

The proposed scheme is demonstrated using PIC simulation code VORPAL [17]. A circularly polarized laser pulse of wavelength $\lambda=0.8 \mu \mathrm{m}$, peak intensity $I_{0}=$ $2.14 \times 10^{23} \mathrm{~W} / \mathrm{cm}^{2}$, corresponding to a laser parameter $a=e A / m_{e} c^{2}=316 / \sqrt{2}$, where $A$ is the vector potential, $c$ is the light speed in vacuum, and $m_{e}$ and $e$ are the electron mass and charge, respectively. The pulse width is $12.5 \lambda$, and the full width at half maximum spot size is $20 \lambda$ (corresponding to a total energy of about $18.26 \mathrm{~kJ}$ ), enters the left side of the $50 \lambda \times 200 \lambda$ simulation box. The background plasma is of density $n_{0}=0.3 n_{c}$, where $n_{c}$ is the critical density, and bounded sharply in $10 \lambda<x<800 \lambda$, $-50 \lambda<y<50 \lambda$. The microtarget is of density $10 n_{c}$ and bounded in $100 \lambda<x<102 \lambda,-1 \lambda<y<1 \lambda$. The ions in the background plasma and the dense microtarget are singly charged tritium ions and protons, respectively. The slow-moving massive background tritium ions allow the formation of a stable electron bubble with a large spacecharge field [16]. A moving window with $2000 \times 400$ cells is used in the simulations and the particle number per cell for the electrons, protons, and tritium ions is 10 [17]. The longitudinal and transverse resolutions are determined by the laser and plasma wavelengths, respectively. In test cases it is verified that the results are not much affected if the resolution is increased (up to double of that used here). Similarly, the sharp boundaries and uniformity of the background and target plasmas have been chosen mainly for convenience and saving of computational resource. The choice is not crucial for the present problem since the actual configuration will be self-consistently determined as soon as the intense short-pulse laser enters the plasma [17].

Figure 1(a) shows the momentum-vector distribution of the background electrons, where $T$ is the laser period. We can see that most electrons ahead of the bubble are pushed forward by the laser pulse and some electrons return along the bubble boundary. For clarity, the momentum is on the logarithmic scale and the magnitude of the vectors has been magnified 10 times. The electrons ahead of the bubble are strongly compressed by the laser pulse in the axial direction, so that the local plasma in front of the laser becomes opaque. The intense space-charge field of the wake bubble can trap the microtarget protons that have been driven to sufficiently high speeds by the laser-pulse radiation pressure $[9,10]$. As can be seen in Figs. 1(b)-1(d), the trapped protons have a small divergence angle $(\Delta \theta \sim$ $2 \times 10^{-2} \pi \mathrm{rad}$ ) and thus good collimation. This effect can
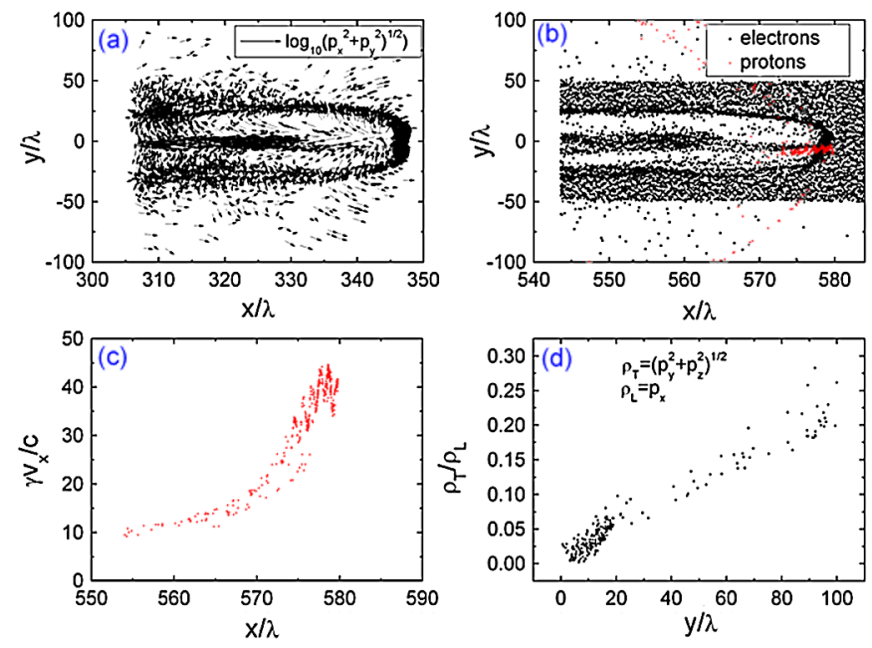

FIG. 1. (Color) (a) Distribution of the momentum vector of the background electrons at $t=360 T$. (b) The background electron and microtarget proton density distributions at $t=600 T$. (c) The proton momentum spectrum at $t=600 T$. (d) The transverse distribution of $\rho_{L} / \rho_{T}$ at $t=800 T$, where $y$ is the distance from the bubble center, and $\rho_{L}=\gamma_{p} m_{p} v_{p x}$ and $\rho_{T}=$ $\gamma_{p} m_{p}\left(v_{p y}^{2}+v_{p z}^{2}\right)^{1 / 2}$ are the longitudinal and transverse momentum of the trapped protons.

be attributed to the transverse force induced by the fastmoving electrons ahead of the bubble, as will be discussed later. As expected, some electrons that have entered the bubble are also trapped and accelerated. However, they are not the main focus of this work and will not be further discussed. The accelerating trapped protons have a sinusoidal structure, which was explained by Nemeth et al. [18] in terms of betatron oscillations. The protons thus trapped by the bubble field can be accelerated stably for a long distance until they overtake the compressed electron layer in front of the bubble. Thus, a quasimonoenergetic proton bunch is produced, as shown in Fig. 2. Most protons in the bunch have an energy of about $38 \mathrm{GeV}$ and the energy

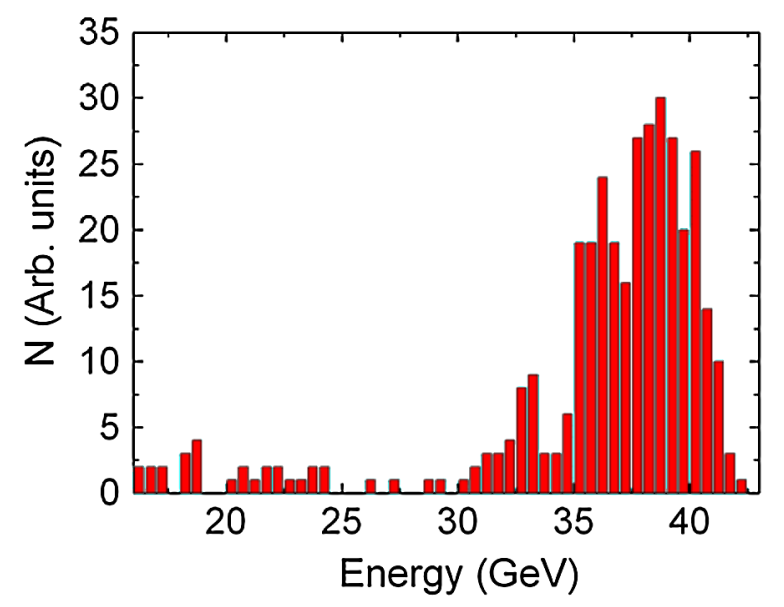

FIG. 2. (Color) Energy spectrum of protons from the simulation at $t=800 T$. 


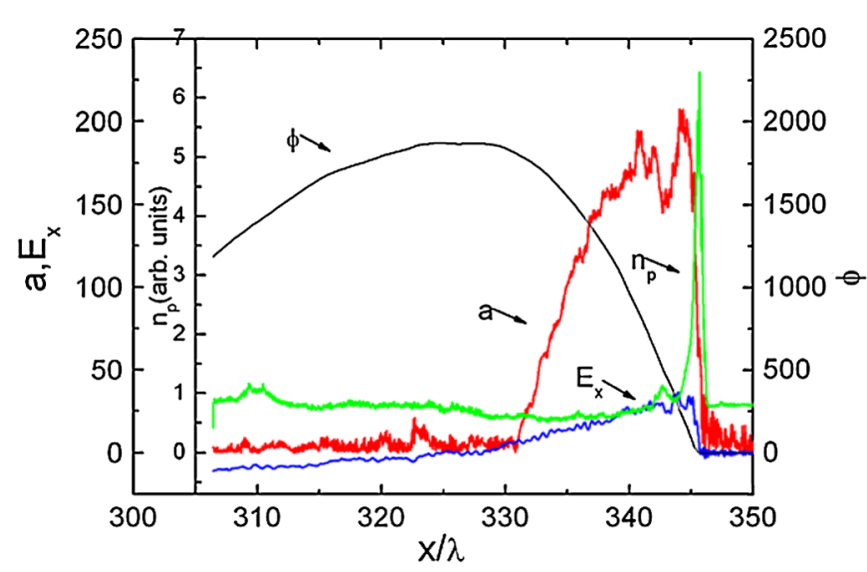

FIG. 3. (Color) The laser amplitude, electrostatic field, electron density, and scalar potential at $t=360 T$.

spread is $\sim 12.6 \%$. The maximum proton energy is almost $42 \mathrm{GeV}$. Higher-quality proton bunches can be obtained if the interaction parameters are such that more protons can be trapped and overtake the bubble, and/or when new schemes for increasing the bubble speed become available.

From conservation of the proton energy $h_{0}=$ $\sqrt{1+p_{x}^{2}+\rho_{p}^{2} a^{2}}-\rho_{p} \phi-v_{p} p_{x}=$ const, where $\rho_{p}=$ $-1 / 1836, p_{x}$ is the proton longitudinal momentum, $\phi$ is the normalized scalar potential, and $v_{p}$ and $\gamma_{p}$ are the bubble velocity and relativistic factor, respectively, one finds the trapping condition $h_{0}+\rho_{p} \phi=1 / \gamma_{p}$ [16]. By recording the positions of the peak electron density in front of the bubble at $t=360 T$ and $600 T$, we find that the average velocity of the bubble in this period is about $v_{p}=$ $0.972 c$, or $\gamma_{p}=4.26$. Thus, a scalar potential $\phi=1405$ is needed to trap a proton initially at rest. Therefore, in our scheme a potential less than 1405 is needed to trap the protons with an initial positive velocity. In Fig. 3, the profiles of the vector potential of the laser, wakefield scalar potential, longitudinal field, and electron density are shown. The maximum normalized scalar potential is about $\phi=1800$, which is sufficiently large to trap a large number of, especially fast, protons. The maximum energy of the trapped and accelerated protons is $W_{\max }=2 \gamma_{p}^{2} m_{p} c^{2} \sim$ $34 \mathrm{GeV}$, which is slightly smaller than that found in our simulation (Fig. 2). The discrepancy can be attributed to the fact that the bubble velocity used in our theoretical estimate is an average velocity.

\section{DISCUSSION}

In order to isolate the effect of the transverse spacecharge field, we show in Fig. 4(a) the electrostatic field $E_{y}$ for the case of a $p$-polarized laser pulse. The other parameters are the same as in Fig. 1. The resulting space-charge field points towards the $x$ axis near the fast-moving electrons and it direction is reversed away from the fast-
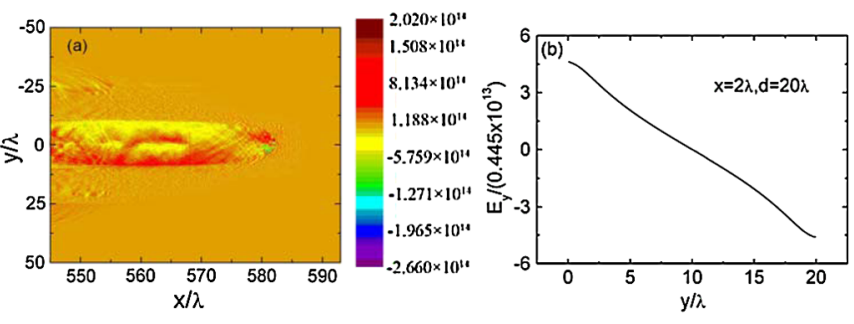

FIG. 4. (Color) (a) Simulation results at $t=600 T$ for the electrostatic field $E_{y}$ for the case of a $p$-polarized laser pulse under the same conditions as that in Figs. 1-3. (b) Analytical result for the transverse electrostatic field $E_{y}$ (in the moving bubble frame) induced by the fast-moving electrons, for the case of the circularly polarized laser considered in Figs. 1-3.

moving electrons, resulting in excellent transverse confinement of the trapped protons. We note that the transverse space-charge field produced by the fast-moving front electron layer points towards the $x$ axis, and that produced by the electrons above and below the bubble points away from the $x$ axis. Thus, near the front electron layer the transverse space-charge field is radially inward, but elsewhere the direction is reversed. Returning to the case of the circularly polarized laser pulse, the transverse electrostatic field induced by the fast-moving electrons in the moving bubble frame is given by

$$
\begin{aligned}
E_{y}= & \frac{e \rho_{s}}{4 \pi \varepsilon_{0}}\left\{\ln \left[x^{2}+(d-y)^{2}\right]-\ln \left(x^{2}+y^{2}\right)\right\} \\
= & 0.445 \times 10^{13}\left\{\ln \left[x^{2}+(d-y)^{2}\right]\right. \\
& \left.-\ln \left(x^{2}+y^{2}\right)\right\}[\mathrm{V} / \mathrm{m}],
\end{aligned}
$$

where $x$ is the distance from the thin electron layer, $d$ is the transverse size, and $\rho_{s}=n_{p} \Delta l=3.09375 \times 10^{21} \mathrm{~m}^{-2}$ the areal density of the thin layer. We have also used $n_{p} \sim$ $6 n_{0}$ and $\Delta l \sim 1 \mu \mathrm{m}$, as obtained from Fig. 3. The variation of $E_{y} /\left(0.445 \times 10^{13}\right)$ along the $y$ axis for $x=2 \lambda$ and $d=$ $20 \lambda$ is shown in Fig. 4(b). We can see that the electrostatic field near the thin electron layer points towards the $x$ axis and is zero at the center. Applying Lorentz transformation, we find that the electrostatic field in the laboratory frame is $E_{y}^{\prime}=\gamma_{p} E_{y}$, where $\gamma_{p}=4.26$, or $E_{y \max }^{\prime}=0.853 \times$ $10^{14} \mathrm{~V} / \mathrm{m}$, agreeing well with the simulation result shown in Fig. 4(a). That is, the fast-moving electrons that generate the transverse electrostatic field are important in the trapping of protons in the transverse direction.

For completeness, Fig. 5(a) shows the self-generated magnetic field $B_{z}$ for the case of the linearly polarized laser pulse at $t=600 T$. As shown in Fig. 5(b), the magnetic field has a maximum of $B_{z} \sim 2.8 \times 10^{5} \mathrm{~T}$ and is quasistatic. Its effect on the protons is roughly opposite to that of the electrostatic field $E_{y}$. Although the Lorentz force exerted by the magnetic field on the protons is in general away from the $x$ axis, for the cases considered the total transverse force acting on protons near the fast- 

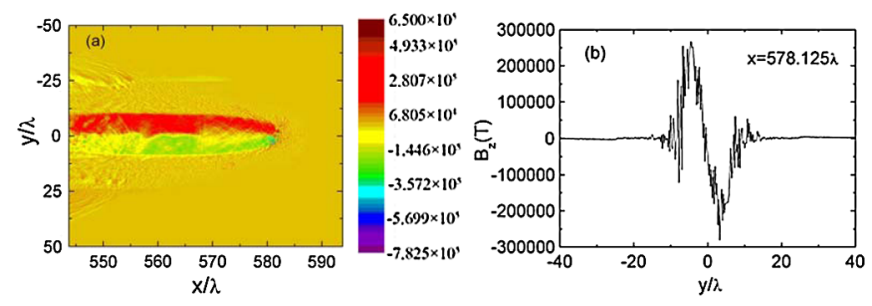

FIG. 5. (Color) (a) Simulation results at $t=600 T$ for the quasistatic self-generated magnetic field $B_{z}$ for the case of a linearly polarized laser pulse. (b) Distribution of the magnetic field $B_{z}$ at $x=578.125 \lambda$ at $t=600 T$.

moving electron layer is still towards the $x$ axis. The resultant transverse force acting on the paraxial protons close to the fast-moving electrons is towards the $x$ axis since the effect of the fast-moving electron layer dominates.

\section{CONCLUSION}

In conclusion, an efficient scheme for obtaining quasimonoenergetic protons of high quality from a hydrogen microtarget in the bubble regime of the interaction of a circularly polarized laser pulse with a tritium plasma is investigated by using 2D PIC simulation. The protons are easily trapped by the wakefield after they have been given an initial velocity by the laser radiation pressure. By varying the microtarget position, density, and size, etc., the quality of the accelerated protons can be controlled. This mechanism can also be effective for lower laser intensities, although at the cost of higher energy spread since the laser depletion length is shorter and a higher background-plasma density is needed to generate a wakefield large enough to trap the protons. For example, a maximum proton energy of about $8 \mathrm{GeV}$ can be obtained with a laser of $a=$ $100 / \sqrt{2}$ and a $3 \lambda \times 3 \lambda$ microtarget in a background plasma of $0.35 n_{c}$. The acceleration effect is found to be not as good with a linearly polarized laser because of appearance of unfavorable instabilities. Furthermore, in real applications the 2D results here should be treated with caution since three-dimensional (3D) effects such as shorter laser Rayleigh length and less-intense space-charge field can be expected to influence the proton acceleration process [19-21].

The efficiency of our scheme appears to be lower than that of proton acceleration using a thin overdense foil [2225]. However, the acceleration process here is more stable, so that tens $\mathrm{GeV}$ protons can be obtained because of the increased acceleration distance. We recall that in the classical radiation-pressure acceleration, which also involves a relatively long acceleration distance, the ion energy scales with $t^{1 / 3}$ or $x^{1 / 3}$ [10], so that it is rather difficult to produce tens $\mathrm{GeV}$ protons. With our microtarget scheme, the highdensity target protons are first accelerated in the usual radiation-pressure regime $[9,10]$ and given sufficiently high initial speeds that allow them to be trapped much more easily by the acceleration field of the wake bubble. When the overdense target is eventually destroyed the trapped protons are further accelerated, but now in the bubble-acceleration regime. Accordingly, the properties of the resulting proton bunch can be controlled by adjusting the background plasma as well as the microtarget densities.

The present scheme differs from that in Refs. [19,26], where the ions are accelerated by the space-charge field created during relativistic laser channeling through an underdense plasma. There the laser intensity must be larger than a threshold value depending on the ratio between ion and electron masses $[19,26]$. Our scheme also differs from ion-wakefield acceleration [27,28], which occurs when the plasma is relativistically transparent to the laser pulse and energetic ions are trapped and accelerated in a plasma wave structure. In that case, there is also a threshold for the laser intensity for ions to be trapped. In our scheme, the protons in the dense microtarget are first accelerated by the laser pressure and then by the wakefield bubble. As a result, a relatively dense bunch of protons can be accelerated stably by the latter for a rather long distance and tens of $\mathrm{GeV}$ protons can be obtained. Since here two acceleration regimes are involved, besides the laser-intensity threshold of Refs. $[19,26]$, the target protons have also to be trapped by the bubble field. But the latter condition is readily satisfied since, as expected because the same laser pulse is responsible for both acceleration regimes of the same protons, the two regimes are normally spatially and temporally joined. Finally, it may be of interest to note that the laser intensity and spot size needed for our scheme may soon be available [29].

\section{ACKNOWLEDGMENTS}

This work is supported by the 973 Program (2006CB806004), the National Natural Science Foundation of China $(10675155,10834008,60921004$, and 10835003), The CAS program (0801051-X00), and the Program of Shanghai Subject Chief Scientists (09XD1404300).

[1] H. Schwoerer, S. Pfotenhauer1, O. Jaeckel, K.-U. Amthor, B. Liesfeld, W. Ziegler, R. Sauerbrey, K. W. D. Ledingham, and T. Esirkepov, Nature (London) 439, 445 (2006).

[2] B. M. Hegelich, B. J. Albright, J. Cobble, K. Flippo, S. Letzring, M. Paffett, H. Ruhl, J. Schreiber, R. K. Schulze, and J. C. Fernandez, Nature (London) 439, 441 (2006).

[3] J. Faure, C. Rechatin, A. Norlin, A. Lifschitz, Y. Glinec, and V. Malka, Nature (London) 444, 737 (2006).

[4] S. P. Hatchett et al., Phys. Plasmas 7, 2076 (2000).

[5] P. Mora, Phys. Rev. Lett. 90, 185002 (2003).

[6] A. Pukhov, Phys. Rev. Lett. 86, 3562 (2001). 
[7] R. A. Snavely et al., Phys. Rev. Lett. 85, 2945 (2000).

[8] D. Habs et al., Prog. Part. Nucl. Phys. 46, 375 (2001).

[9] B. Shen et al., Phys. Rev. E 64, 056406 (2001).

[10] T. Esirkepov et al., Phys. Rev. Lett. 92, 175003 (2004).

[11] M. Chen et al., Phys. Plasmas 15, 113103 (2008).

[12] S. P. D. Mangles et al., Nature (London) 431, 535 (2004); G. G. R. Geddes et al., Nature (London) 431, 538 (2004); J. Faure et al., Nature (London) 431, 541 (2004).

[13] D. Umstadter, J. K. Kim, and E. Dodd, Phys. Rev. Lett. 76, 2073 (1996); E. Esarey et al., Phys. Rev. Lett. 79, 2682 (1997); J. Faure et al., Nature (London) 444, 737 (2006).

[14] S. V. Bulanov et al., Phys. Rev. E 58, R5257 (1998); H. Suk et al., Phys. Rev. Lett. 86, 1011 (2001); T.-Y. Chien et al., Phys. Rev. Lett. 94, 115003 (2005); A. V. Brantov, T. Zh. Esirkepov, M. Kando, H. Kotaki, V. Yu. Bychenkov, and S. V. Bulanov, Phys. Plasmas 15, 073111 (2008).

[15] B. Shen, Y. Li, K. Nemeth, H. Shang, Y. Chae, R. Soliday, R. Crowell, E. Frank, W. Gropp, and J. Cary, Phys. Plasmas 14, 053115 (2007).

[16] B. Shen, Y. Li, M. Y. Yu, and J. Cary, Phys. Rev. E 76, 055402(R) (2007).

[17] C. Nieter and J. R. Cary, J. Comput. Phys. 196, 448 (2004).

[18] K. Nemeth, B. Shen, Y. Lin, H. Shang, R. Crowell, K. C. Harkay, and J. R. Cary, Phys. Rev. Lett. 100, 095002
(2008).

[19] F. Pegoraro et al., IEEE Trans. Plasma Sci. 28, 1177 (2000).

[20] A. Pukhov, Rep. Prog. Phys. 66, 47 (2003).

[21] W. Lu et al., Phys. Rev. ST Accel. Beams 10, 061301 (2007).

[22] A. Macchi, F. Cattani, T. V. Liseykina, and F. Cornolti, Phys. Rev. Lett. 94, 165003 (2005).

[23] O. Klimo, J. Psikal, J. Limpouch, and V. T. Tikhonchuk, Phys. Rev. ST Accel. Beams 11, 031301 (2008).

[24] A. P. L. Robinson, M. Zepf, S. Kar, R. G. Evans, and C. Bellei, New J. Phys. 10, 013021 (2008); A. P. L. Robinson, P. Gibbon, M. Zepf, S. Kar, R. G. Evans, and C. Bellei, Plasma Phys. Controlled Fusion 51, 024004 (2009).

[25] X. Q. Yan, C. Lin, Z. M. Sheng, Z. Y. Guo, B. C. Liu, Y. R. Lu, J.X. Fang, and J.E. Chen, Phys. Rev. Lett. 100, 135003 (2008).

[26] Y. Sentoku et al., Phys. Rev. E 62, 7271 (2000).

[27] O. Shorokhov and A. Pukhov, Laser Part. Beams 22, 175 (2004).

[28] A. Pukhov et al., Nucl. Fusion 44, S191 (2004).

[29] See, for example, http://www.extreme-lightinfrastructure.eu. 